\title{
Effect of Dietary Inclusion of Lemon Balm (Melissa Officinalis L.) Extract on Performance, Gut Microflora, Blood Parameters, Immunity and Carcass Traits of Broilers
}

\author{
Monireh Poorghasemi ${ }^{1}$, Alireza Seidavi ${ }^{2}$, Mehrdad Mohammadi ${ }^{1}$, \\ João Simões ${ }^{3}$, Vito Laudadio ${ }^{4}$ and Vincenzo Tufarelli ${ }^{4}$ \\ ${ }^{1}$ Department of Animal Science, College of Agriculture, University of Guilan, Rasht, Iran \\ ${ }^{2}$ Department of Animal Science, Rasht Branch, Islamic Azad University, Rasht, Iran \\ ${ }^{3}$ Department of Veterinary Science, University of Trás-os-Montes e Alto Douro, Quinta de prados, Vila Real, Portugal \\ ${ }^{4}$ Department of DETO, Section of Veterinary Science and Animal Production, \\ University of Bari ‘Aldo Moro', Valenzano, Bari, Italy
}

\begin{abstract}
The aim of the present study was to evaluate the effects of lemon balm (LB; Melissa officinalis L.) extract as additive on performance, health status and carcass traits of broilers during a 42-days production cycle. One hundred mixed chicks of Ross 308 strain were assigned for five dietary treatments with four replicates per group and five birds per replicate as follows: control diet, 0.5LB, 1.0LB, $1.5 \mathrm{LB}$ and $2.0 \mathrm{LB}$ with $0.0,0.5,1.0,1.5$ or $2.0 \mathrm{~mL}$ of $\mathrm{LB}$ extract per liter of drinking water, respectively. In overall, at 42nd day, low feed, energy and protein efficiency $(P<0.05)$ were observed in 1.0LB group than in control diet. However, during the 3rd and 5-6th weeks, feed, energy and protein intakes were improved $(P<0.05)$, without any efficiency enhancement $(P>0.05)$ mainly in group on 1.0LB diet. During the 5 th week of rearing, daily weight gain was higher $(P<0.05)$ in groups $0.5 \mathrm{LB}, 1.0 \mathrm{LB}$ and $2.0 \mathrm{LB}$ compared to control diet. At the end of feeding period, cecal enterococcus bacteria colony count was higher $(P<$ $0.05)$ and left cecum diameter was lower $(P<0.05)$ in 1.0LB group. Hematological parameters and viscera and carcass traits remained unaffected $(P>0.05)$ by dietary treatments. In conclusion, the supplementation with LB as natural feed additive resulted in a potential positive effect on broilers performance mainly during the grower and finisher periods.
\end{abstract}

Key words: broiler, growth, lemon balm, microbiota, performance

\section{Introduction}

Lemon balm (Melissa officinalis L.; LB) is a perennial herb that grows wildly in Mediterranean region and southcentral Europe, and it is also successfully cultivated in few countries such as the agricultural research organizations in Taiwan (Lin et al., 2012). In human, LB is widely used as culinary, aromatic and medicinal herb (Kato-Noguchi, 2003), in this last case in both traditional and alternative medicine (Khan et al., 2012; Dhama et al., 2015; Alzawqari

Received: January 4, 2017, Accepted: January 31, 2017

Released Online Advance Publication: April 25, 2017

Correspondence: Dr. Alireza Seidavi, Department of Animal Science, Rasht Branch, Islamic Azad University, Rasht, Iran.

(E-mail: alirezaseidavi@iaurasht.ac.ir), and

Dr.Vincenzo Tufarelli, Department of DETO, Section of Veterinary Science and Animal Production, University of Bari 'Aldo Moro', Valenzano, Bari, Italy. (E-mail: vincenzo.tufarelli@uniba.it) et al., 2016).

The major active compounds present on commercial LB plants are flavonoids such as gallic acid, catechin, chlorogenic acid, caffeic acid, ellagic acid, epicatechin, rutin and quercetin, having antimicrobial and strong antioxidant activities (Mimica-Dukic et al., 2004; Pereira et al., 2014). The use of LB as feed additive in broiler diet, in order to improve meat quality (Marcinčák et al., 2011a, b) or health, and to substitute antibiotics as growth promoter has been recently reported in few studies, even in organic poultry production (Eleroğlu et al., 2013, 2014). A higher relative weight of leg and breast muscles and polyunsaturated fatty acids percentage in meat were observed in broilers supplemented with LB extract by Marcinčáková et al. (2011a) confirming its antioxidant activity. Antioxidant effects without performance changes of a clove and LB mixture were also observed by Petrovic et al. (2012). Similar observations on performance were also reported by Marcinčák et al. 
(2011b). However, a deep research considering LB use as single feed additive at different dietary inclusion levels was not yet reported. In fact, little research exists evaluating simultaneous both performance and physiologic status of commercial broilers fed LB extract in drinking. Therefore, the objective of this study was to determine the effect of different levels of LB extract in drinking water to broiler chicks performance, immunity, blood constituents, carcass characteristics and gut microflora from hatch to slaughtering age.

\section{Materials and Methods}

\section{Animals, Experimental Treatments and Diet}

The experiment was performed housing a total of 100 mixed chicks of the Ross 308 strain (Aviagen, Newbridge, Scotland, UK 35805) in a poultry facility at the University of Guilan, Rasht, Iran and at the Faculty of Agriculture, Islamic Azad University, Rasht Branch, Rasht, Iran. The facility was $12 \times 25$ meters with four ventilators and 6 windows, and a total 20 land-pens $(1.6 \times 0.8 \mathrm{~m})$ were used. Ventilation was turned on to optimize the climate $24 \mathrm{~h}$ before the broilers were brought in. A heater was used and temperature program was according to the instructions for Ross 308 broilers (Aviagen, Newbridge, Scotland, UK 35805). Air humidity was kept at 55 to $65 \%$ in the early growing period by spraying water on the floor. Lamps were installed at a height of 2.2 meters above the floor. Twenty-three hours lighting was on and daily for one hour between 19:00 and 20:00 the house was left dark till slaughter at day 42. Sanitation principles and health measures for raising chickens were applied.

The experimental design included five dietary treatments with four replicates per treatment. One-day-old chicks were allotted to 20 groups of five birds, such that mean group body weight (BW) was similar for each group. A 12 (female) to 8 (male) ratio for female to male was the same for all treatments. Environmental conditions were also similar for all treatments.

The dietary treatments were as follows: Treatment 1 as control diet with water included no LB extract $(0 \mathrm{~mL} / \mathrm{L})$; Treatment 2 (0.5LB: water included LB extract at $0.5 \mathrm{~mL} / \mathrm{L})$; Treatment 3 (1.0LB): water included LB extract at 1.0 $\mathrm{mL} / \mathrm{L})$; Treatment 4 (1.5LB): water included LB extract at $1.5 \mathrm{~mL} / \mathrm{L})$ and Treatment 5 (2.0LB): water included LB extract at $2.0 \mathrm{~mL} / \mathrm{L}$ ).

All chickens were fed according to the producer's feeding instructions. The composition of diets and their nutrient composition during the starter (1st-14th day of age), grower (15th-28th day of age) and finisher (29th-42nd day of age) periods are given in Table 1.

The LB was acquired by Agricultural-Jihad Organization of Hamadan, Hamadan, Iran and the extract was prepared as described by Bombik et al. (2012). A total of $200 \mathrm{~g}$ of dried LB per liter of drinking water was daily infused for $10 \mathrm{~min}$, cooled to $40^{\circ} \mathrm{C}$ and then strained.

\section{Performance Parameters}

Daily feed intake (g/day) and BW gain (g/day) were determined weekly. Feed efficiency (total feed intake / total
BW gain), energy intake (kcal/day), energy efficiency (energy intake / BW gain), daily protein intake (g/day), protein efficiency (protein intake/ BW gain) and production index [total BW $\times$ livability $(\%) /$ (age days $\times$ feed conversion ratio $\times 10)$ ] were calculated.

\section{Immunity Traits}

Birds were vaccinated against prevalence diseases. After each vaccination, 1:1000 multivitamin + electrolytes solution was mixed in the drinking water for $24 \mathrm{~h}$. Feed remaining in feeders, after each time was weighed at the end of the week. Vaccination against Avian Influenza disease virus (Avian Influenza- H9N2; Razi Co, Karaj, Iran) in one administration of 10-day old were taken and humoral immune response of chickens to the vaccine at 35-day and 42-day old was sampled and measured based on haemagglutination inhibition method. Meanwhile, vaccination against Infectious Bronchitis disease virus (Infectious Bronchitis Virus -H120; Razi Co, Karaj, Iran) in two administrations of 1-days and 15days old were taken and humoral immune response of chickens to the vaccine at 25-day old was sampled and measured based on ELISA method (IDEXX Laboratories, B. V., The Netherlands) according to the manufacturer instructions.

\section{Hematology Traits}

At 42 days of age, a $5 \mathrm{~mL}$ volume of venous blood was collected from the ulnaris vein in the wing of one bird taken from each replicate. Care was taken to choose the most representative male birds with respect to body weight compared to the group mean body weight. Before blood collections were made, feed was removed from all the birds for a period of four hours in an attempt to allow stabilization of the various plasma constituents, and all blood sampling was done in the morning to further reduce the variability of the plasma constituents to be measured.

The whole blood sample was transferred from the syringe into a tube coated with $10 \mathrm{mg}$ of the anticoagulant ethylene diamine tetra acetic acid (EDTA). Blood samples were centrifuged at $3000 \mathrm{rpm} \times 20 \mathrm{~min}$ to assure separation of the blood cells from the plasma. Plasma was collected and stored at $-20^{\circ} \mathrm{C}$ until plasma constituent analyses (glucose, uric acid, triglycerides, total cholesterol, very low density lipoprotein, high and low density lipoproteins cholesterol, aspartate aminotransferase, alanine aminotransferase, total protein, albumin and globulin) following standard protocols for Roche Cobas Integra 400 Plus analyzer (Roche Diagnostics, GmbH, Mannheim Germany) and commercial kits (Teif Azmoon Pars, Co., Tehran, Iran), according to the manufacturer instructions.

\section{Viscera and Carcass Traits}

At the age of 42 days, after 4 hours of fasting for complete evacuation of the gut, the selected birds were euthanized and used for measuring carcass yield and distribution of meat and gastrointestinal tract characteristics. Birds $(n=20)$ were fully pecked by dry pecking method. Feet were separated from the carcass in the tibio-tarsal joint. Neck, wingtips, gut and liver were removed and the empty or edible carcass was weighed and intestinal segments dimensions were recorded. 
Table 1. Ingredients and nutrient analysis of diets fed to broiler chickens

\begin{tabular}{lrrr}
\hline \hline \multicolumn{1}{c}{ Ingredient (\%) } & $\begin{array}{c}\text { Starter } \\
\text { period }\end{array}$ & $\begin{array}{c}\text { Grower } \\
\text { period }\end{array}$ & $\begin{array}{c}\text { Finisher } \\
\text { period }\end{array}$ \\
\hline Corn & 50.53 & 50.96 & 49.34 \\
Soybean Meal & 37.52 & 32.10 & 27.79 \\
Wheat & 5.00 & 10.00 & 15.00 \\
Soybean oil & 2.14 & 2.79 & 3.89 \\
Ca\%22P\%18 & 1.90 & 1.67 & 1.60 \\
NaCl & 0.34 & 0.33 & 0.32 \\
DL-Methionine & 0.26 & 0.27 & 0.25 \\
Lysine-Hydro-Chloride & 0.29 & 0.21 & 0.19 \\
Mineral premix* & 0.25 & 0.25 & 0.25 \\
Vitamin premix** & 0.25 & 0.25 & 0.25 \\
CaCO3 & 1.23 & 1.01 & 0.97 \\
Threonine & 0.09 & 0.06 & 0.05 \\
NaHCO3 & 0.10 & 0.10 & 0.10 \\
Nutrient analysis & & & \\
ME (kcal/kg diet) & 2,900 & 3,000 & 3,100 \\
Crude protein (\%) & 21.00 & 20.00 & 18.50 \\
Calcium (\%) & 1.01 & 0.86 & 0.82 \\
Available P (\%) & 0.48 & 0.43 & 0.41 \\
Potassium (\%) & 0.38 & 0.38 & 0.38 \\
Chloride (\%) & 0.15 & 0.15 & 0.15 \\
Sodium (\%) & 0.15 & 0.15 & 0.15 \\
Arginine (\%) & 1.39 & 1.21 & 0.10 \\
Lysine (\%) & 1.37 & 1.18 & 1.06 \\
Meth + Cys (\%) & 1.03 & 0.90 & 0.83 \\
Threonine (\%) & 0.90 & 0.79 & 0.72 \\
Tryptophan (\%) & 0.23 & 0.19 & 0.17 \\
\hline Calcium Pantor & & & \\
\hline
\end{tabular}

*Calcium Pantothenate: $4 \mathrm{mg} / \mathrm{g}$; Niacin: $15 \mathrm{mg} / \mathrm{g}$; Vitamin B6: $13 \mathrm{mg} / \mathrm{g}$; Cu: 3 $\mathrm{mg} / \mathrm{g} ; \mathrm{Zn}: 15 \mathrm{mg} / \mathrm{g}$; Mn: $20 \mathrm{mg} / \mathrm{g} ; \mathrm{Fe}: 10 \mathrm{mg} / \mathrm{g} ; \mathrm{K}: 0.3 \mathrm{mg} / \mathrm{g}$. **Vitamin A: $5000 \mathrm{IU} / \mathrm{g}$; Vitamin D3: $500 \mathrm{IU} / \mathrm{g}$; Vitamin E: $3 \mathrm{mg} / \mathrm{g}$; Vitamin K3: $1.5 \mathrm{mg} / \mathrm{g}$; Vitamin B2: $1 \mathrm{mg} / \mathrm{g}$.

Breast muscle (including skin and sternum) was dissected from carcass. Legs (including thighs and drumsticks) were dissected by ex-articulation in hip joint and dissecting tissue from iliac bone. Abdominal fat, including that around the rectum, gizzard and proventriculus was collected. The length $(\mathrm{cm})$, width $(\mathrm{mm})$ and wall thickness $(\mathrm{mm})$ of duodenum, ileum jejunum, left and right cecum and colon were recorded. Total weight of all dissected parts and the weights of various segments of the digestive tract were related to the totally eviscerated carcass. Ratios were calculated according to the following formula: [(weight of component(s)/eviscerated carcass weight $) \times 100]$.

\section{Microbiota}

Agar plates were streaked with cecum and gizzard contents of selected birds and sent to the laboratory. To determinate bacterial growth and colony counts, the agar plates streaked on the site were used. Collecting tubes were weighted, wrapped into aluminum sheet and autoclaved for $10 \mathrm{~min}$. The culture mediums were prepared and 24 hours before collecting samples were poured into the petri dish. MRS agar (Man Rogosa Sharpe agar, 1.10660.500) to culture lactic acid producing bacteria, Eosin Metilan Blou
(EMB, 1.01347.0500) to culture Escherichia coli, Macconkey agar (105465.0500) to culture coliforms, Azide dextrose broth (101590) to culture enterococcus bacteria, and Nutrient agar $(1.05450 .0500)$ was used to culture total aerobic bacteria counts, respectively. Total aerobic bacteria and total acid producing bacteria were also accessed.

All procedures were approved by the Authors' Institution's Ethic Committee, and care was taken to minimize the number of animals used.

\section{Statistical Analysis}

Data were analyzed by analysis of variance using a oneway ANOVA procedure based on a completely randomized design. Data were analyzed by $\mathrm{SAS}^{\circledR} 8.0$ statistical software (SAS Institute Inc., Cary, NC, USA) and GLM procedure was used. The means ( \pm SEM) were compared by using Duncan's test. Results were considered significantly different at $P<0.05$.

\section{Results}

\section{Performance Traits}

No differences $(P>0.05)$ between groups on performance traits were observed during the 1 st week of rearing (Table 2). 
Table 2. Performance traits of broilers fed different levels of lemon balm extract according to the rearing period

\begin{tabular}{|c|c|c|c|c|c|c|c|}
\hline Item & $\begin{array}{c}\text { Daily feed } \\
\text { intake }(\mathrm{g})\end{array}$ & $\begin{array}{c}\text { Daily BW } \\
\text { gain }(\mathrm{g})\end{array}$ & $\begin{array}{c}\text { Feed } \\
\text { efficiency }\end{array}$ & $\begin{array}{l}\text { Daily } \\
\text { energy } \\
\text { intake } \\
\text { (kcal) }\end{array}$ & $\begin{array}{c}\text { Energy } \\
\text { efficiency } \\
(\mathrm{kcal} / \mathrm{g})\end{array}$ & $\begin{array}{c}\text { Daily } \\
\text { protein } \\
\text { intake }(\mathrm{g})\end{array}$ & $\begin{array}{l}\text { Protein } \\
\text { Efficiency } \\
\quad(g / g)\end{array}$ \\
\hline & \multicolumn{7}{|c|}{ 1st week (1st - 7th day) } \\
\hline Control & 18.9 & 17.6 & 1.14 & 57.5 & 3.32 & 4.0 & 18.9 \\
\hline $0.5 \mathrm{LB}$ & 20.0 & 17.4 & 1.15 & 58.0 & 3.33 & 4.0 & 20.0 \\
\hline 1.0 LB & 19.6 & 15.6 & 1.26 & 57.0 & 3.67 & 3.9 & 19.6 \\
\hline $1.5 \mathrm{LB}$ & 19.8 & 15.9 & 1.25 & 57.4 & 3.62 & 4.0 & 19.8 \\
\hline $2.0 \mathrm{LB}$ & 21.6 & 17.0 & 1.26 & 62.6 & 3.67 & 4.3 & 21.6 \\
\hline \multirow{2}{*}{$( \pm \mathrm{SEM})$} & $( \pm 0.6)$ & $( \pm 0.5)$ & $( \pm 0.03)$ & $( \pm 1.7)$ & $( \pm 0.07)$ & $( \pm 0.1)$ & $( \pm 0.01)$ \\
\hline & \multicolumn{7}{|c|}{ 2nd week (8th - 14th day) } \\
\hline Control & 46.7 & $38.1^{\mathrm{a}}$ & 1.23 & 137.8 & 3.64 & 9.6 & 0.25 \\
\hline $0.5 \mathrm{LB}$ & 40.5 & $28.6^{\mathrm{b}}$ & 1.42 & 119.5 & 4.19 & 8.3 & 0.29 \\
\hline $1.0 \mathrm{LB}$ & 39.0 & $29.8^{\mathrm{b}}$ & 1.33 & 115.0 & 3.92 & 8.0 & 0.27 \\
\hline 1.5 LB & 37.8 & $30.2^{\mathrm{b}}$ & 1.25 & 111.6 & 3.70 & 7.8 & 0.25 \\
\hline $2.0 \mathrm{LB}$ & 42.5 & $31.1^{\mathrm{ab}}$ & 1.38 & 125.4 & 4.06 & 8.7 & 0.28 \\
\hline \multirow[t]{2}{*}{$( \pm$ SEM $)$} & $( \pm 1.4)$ & $( \pm 1.2)$ & $( \pm 0.04)$ & $( \pm 4.2)$ & $( \pm 0.11)$ & $( \pm 0.3)$ & $( \pm 0.01)$ \\
\hline & \multicolumn{7}{|c|}{$3 r d$ week $(15 t h-21 s t$ day) } \\
\hline Control & $81.1^{\mathrm{a}}$ & $51.6^{\mathrm{ab}}$ & $1.46^{\mathrm{a}}$ & $251.3^{\mathrm{a}}$ & $4.55^{\mathrm{a}}$ & $16.2^{\mathrm{a}}$ & $0.29^{\mathrm{a}}$ \\
\hline $0.5 \mathrm{LB}$ & $92.1^{\mathrm{b}}$ & $55.1^{\mathrm{a}}$ & $1.80^{\mathrm{b}}$ & $285.4^{\mathrm{b}}$ & $5.58^{\mathrm{b}}$ & $18.4^{\mathrm{b}}$ & $0.36^{\mathrm{b}}$ \\
\hline $1.0 \mathrm{LB}$ & $91.7^{\mathrm{b}}$ & $53.9^{\mathrm{ab}}$ & $1.70^{\mathrm{ab}}$ & $284.3^{\mathrm{b}}$ & $5.27^{\mathrm{ab}}$ & $18.3^{\mathrm{b}}$ & $0.34^{\mathrm{ab}}$ \\
\hline $1.5 \mathrm{LB}$ & $78.2^{\mathrm{a}}$ & $50.5^{\mathrm{a}}$ & $1.55^{\mathrm{ab}}$ & $242.3^{\mathrm{a}}$ & $8.41^{\mathrm{ab}}$ & $15.6^{\mathrm{b}}$ & $0.31^{\mathrm{a}}$ \\
\hline \multirow{3}{*}{$\begin{array}{l}2.0 \mathrm{LB} \\
( \pm \mathrm{SEM})\end{array}$} & $92.6^{\mathrm{b}}$ & $59.6^{\mathrm{b}}$ & $1.56^{\mathrm{ab}}$ & $287.4^{\mathrm{b}}$ & $4.83^{\mathrm{ab}}$ & $18.5^{\mathrm{b}}$ & $0.31^{\mathrm{ab}}$ \\
\hline & $( \pm 1.9)$ & $( \pm 1.1)$ & $( \pm 0.04)$ & $( \pm 5.6)$ & $( \pm 0.12)$ & $( \pm 0.4)$ & $( \pm 0.01)$ \\
\hline & \multicolumn{7}{|c|}{ 4th week (22nd - 28th day) } \\
\hline Control & 118.6 & 69.5 & $1.71^{\mathrm{a}}$ & 361.6 & $5.23^{\mathrm{a}}$ & 22.8 & $0.33^{\mathrm{a}}$ \\
\hline $0.5 \mathrm{LB}$ & 119.4 & 82.6 & $1.44^{\mathrm{b}}$ & 364.1 & $4.41^{\mathrm{b}}$ & 23.0 & $0.28^{\mathrm{b}}$ \\
\hline $1.0 \mathrm{LB}$ & 122.6 & 68.2 & $1.81^{\mathrm{a}}$ & 373.4 & $5.54^{\mathrm{a}}$ & 23.6 & $0.35^{\mathrm{a}}$ \\
\hline 1.5 LB & 127.3 & 75.0 & $1.70^{\mathrm{a}}$ & 388.1 & $5.19^{\mathrm{a}}$ & 24.5 & $0.32^{\mathrm{a}}$ \\
\hline \multirow{3}{*}{$\begin{array}{l}2.0 \mathrm{LB} \\
( \pm \mathrm{SEM})\end{array}$} & 129.2 & 70.7 & $1.83^{\mathrm{a}}$ & 394.1 & $5.61^{\mathrm{a}}$ & 24.9 & $0.35^{\mathrm{a}}$ \\
\hline & $( \pm 2.6)$ & $( \pm 2.2)$ & $( \pm 0.04)$ & $( \pm 8.0)$ & $( \pm 0.13)$ & $( \pm 0.5)$ & $( \pm 0.01)$ \\
\hline & \multicolumn{7}{|c|}{5 th week (29th - 35th day) } \\
\hline Control & $168.8^{\mathrm{a}}$ & $87.0^{\mathrm{a}}$ & 1.94 & $523.4^{\mathrm{a}}$ & 6.03 & $31.2^{\mathrm{a}}$ & 0.36 \\
\hline $0.5 \mathrm{LB}$ & $173.9^{\mathrm{ab}}$ & $96.0^{\mathrm{b}}$ & 1.81 & $539.0^{\mathrm{ab}}$ & 5.61 & $32.2^{\mathrm{ab}}$ & 0.33 \\
\hline $1.0 \mathrm{LB}$ & $184.7^{\mathrm{b}}$ & $95.9^{\mathrm{b}}$ & 1.92 & $572.6^{\mathrm{b}}$ & 5.98 & $34.2^{\mathrm{b}}$ & 0.35 \\
\hline 1.5 LB & $172.1^{\mathrm{ab}}$ & $92.1^{\mathrm{ab}}$ & 1.88 & $533.6^{\mathrm{ab}}$ & 5.82 & $31.8^{\mathrm{ab}}$ & 0.34 \\
\hline \multirow{3}{*}{$\begin{array}{l}2.0 \mathrm{LB} \\
( \pm \mathrm{SEM})\end{array}$} & $176.9^{\mathrm{ab}}$ & $96.0^{\mathrm{b}}$ & 1.84 & $548.5^{\mathrm{ab}}$ & 5.71 & $23.7^{\mathrm{ab}}$ & 0.34 \\
\hline & $( \pm 2.1)$ & $( \pm 1.3)$ & $( \pm 0.02)$ & $( \pm 6.6)$ & $( \pm 0.07)$ & $( \pm 0.4)$ & $( \pm 0.01)$ \\
\hline & \multicolumn{7}{|c|}{ 6th week (36th-42nd day) } \\
\hline Control & $188.1^{\mathrm{a}}$ & 99.8 & 1.88 & $583.1^{\mathrm{a}}$ & 5.85 & $34.8^{\mathrm{a}}$ & 0.34 \\
\hline $0.5 \mathrm{LB}$ & $202.1^{\mathrm{ab}}$ & 97.0 & 2.08 & $626.6^{\mathrm{ab}}$ & 6.46 & $37.4^{\mathrm{ab}}$ & 0.38 \\
\hline $1.0 \mathrm{LB}$ & $209.0^{\mathrm{b}}$ & 90.0 & 2.13 & $647.8^{\mathrm{b}}$ & 6.61 & $38.7^{\mathrm{b}}$ & 0.39 \\
\hline $1.5 \mathrm{LB}$ & $190.0^{\mathrm{a}}$ & 96.7 & 2.03 & $589.0^{\mathrm{a}}$ & 6.29 & $35.1^{\mathrm{a}}$ & 0.37 \\
\hline $2.0 \mathrm{LB}$ & $191.4^{\mathrm{a}}$ & 117.4 & 1.71 & $593.3^{\mathrm{a}}$ & 5.30 & $35.4^{\mathrm{a}}$ & 0.31 \\
\hline$( \pm \mathrm{SEM})$ & $( \pm 2.8)$ & $( \pm 4.1)$ & $( \pm 0.06)$ & $( \pm 8.7)$ & $( \pm 0.19)$ & $( \pm 0.5)$ & $( \pm 0.01)$ \\
\hline
\end{tabular}

LB - Lemon balm extract.

Different letters within the same column indicate significant differences among treatment groups $(P<0.05)$

During the 2nd week and consequently throughout the starter period, the daily weight gain was lower $(P<0.05)$ in groups $0.5 \mathrm{LB}, 1.0 \mathrm{LB}$ and $1.5 \mathrm{LB}$ compared to control group. During the 3rd week, daily feed, energy and protein intakes were higher $(P<0.05)$ in groups $0.5 \mathrm{LB}$ and $2.0 \mathrm{LB}$ than in control. However, and contrarily to the 4 th week, poorly feed, energy 
Table 3. Performance mean ( \pm SEM) of Ross 308 broilers fed the different levels of lemon balm at the 42-day production cycle

\begin{tabular}{lccccccccc}
\hline \hline Item & $\begin{array}{c}\text { Daily feed } \\
\text { intake (g) }\end{array}$ & $\begin{array}{c}\text { Daily BW } \\
\text { gain (g) }\end{array}$ & $\begin{array}{c}\text { Feed } \\
\text { efficiency }\end{array}$ & $\begin{array}{c}\text { Daily } \\
\text { energy } \\
\text { intake } \\
(\mathrm{kcal})\end{array}$ & $\begin{array}{c}\text { Energy } \\
\text { efficiency } \\
(\mathrm{kcal} / \mathrm{g})\end{array}$ & $\begin{array}{c}\text { Daily } \\
\text { protein } \\
\text { intake } \\
(\mathrm{g})\end{array}$ & $\begin{array}{c}\text { Protein } \\
\text { Efficiency } \\
(\mathrm{g} / \mathrm{g})\end{array}$ & $\begin{array}{c}\text { Live BW } \\
(\mathrm{g})\end{array}$ & $\begin{array}{c}\text { Production } \\
\text { index }\end{array}$ \\
\hline Control & 103.9 & 61.2 & $1.69^{\mathrm{a}}$ & 311.6 & $5.09^{\mathrm{a}}$ & 20.6 & $0.35^{\mathrm{a}}$ & 2553 & 358.5 \\
$0.5 \mathrm{LB}$ & 108.0 & 62.1 & $1.73^{\mathrm{ab}}$ & 324.0 & $5.21^{\mathrm{ab}}$ & 21.4 & $0.34^{\mathrm{ab}}$ & 2665 & 365.0 \\
$1.0 \mathrm{LB}$ & 111.1 & 60.3 & $1.84^{\mathrm{b}}$ & 333.3 & $5.54^{\mathrm{b}}$ & 20.0 & $0.37^{\mathrm{b}}$ & 2.507 & 324.2 \\
$1.5 \mathrm{LB}$ & 104.2 & 60.6 & $1.73^{\mathrm{ab}}$ & 312.6 & $5.21^{\mathrm{ab}}$ & 20.7 & $0.34^{\mathrm{ab}}$ & 2568 & 355.8 \\
$2.0 \mathrm{LB}$ & 109.0 & 65.3 & $1.67^{\mathrm{a}}$ & 327.1 & $5.02^{\mathrm{a}}$ & 21.6 & $0.33^{\mathrm{a}}$ & 2734 & 391.7 \\
$( \pm$ SEM $)$ & $( \pm 1.2)$ & $( \pm 0.9)$ & $( \pm 0.02)$ & $( \pm 3.5)$ & $( \pm 0.06)$ & $( \pm 0.22)$ & $( \pm 0.01)$ & $( \pm 44)$ & $( \pm 9.6)$ \\
\hline
\end{tabular}

LB - Lemon balm extract.

Different letters within the same column indicate significant differences among treatment groups $(P<0.05)$.

Table 4. Effect of different levels of lemon balm extract into drinking water on gizzard and cecum microflora $(\log 10 \mathrm{CFU} / \mathrm{g})$ of broilers at 42nd day of age

\begin{tabular}{ccccc}
\hline \hline \multirow{2}{*}{ Item } & \multicolumn{2}{c}{ Gizzard } & & Cecum \\
\cline { 2 - 3 } Control & Coliforms & Escherichia coli & & Enterococcus \\
\hline $0.5 \mathrm{LB}$ & $8.53^{\mathrm{a}}$ & $7.89^{\mathrm{ab}}$ & $6.62^{\mathrm{a}}$ \\
$1.0 \mathrm{LB}$ & $8.66^{\mathrm{b}}$ & $7.99^{\mathrm{a}}$ & $6.94^{\mathrm{ab}}$ \\
$1.5 \mathrm{LB}$ & $8.49^{\mathrm{a}}$ & $7.87^{\mathrm{ab}}$ & $7.16^{\mathrm{b}}$ \\
$2.0 \mathrm{LB}$ & $8.69^{\mathrm{b}}$ & $7.87^{\mathrm{ab}}$ & $7.09^{\mathrm{b}}$ \\
\pm SEM & $8.54^{\mathrm{a}}$ & $7.70^{\mathrm{b}}$ & & $7.09^{\mathrm{b}}$ \\
\hline
\end{tabular}

LB - Lemon balm extract.

Different letters within the same column indicate significant differences among treatment groups $(P<0.05)$.

and protein efficiencies $(P<0.05)$ were observed in group $0.5 \mathrm{LB}$. Considering the whole grower period, low performance efficiency $(P<0.05)$ was observed only in group fed 1.0 LB. During the 5th and 6th weeks, the daily feed, energy and protein intakes were higher $(P<0.05)$ in group 1.0LB than control group, without $(P>0.05)$ feed, energy or protein efficiencies degradation.

In overall, considering the whole production cycle (42 days; Table 3), the group 1.0LB showed poorly feed, energy and protein efficiency $(P<0.05)$ than control group or group 2.0LB, without daily weight gain improvement. No other significant enhancements $(P>0.05)$ were observed in the remained groups.

\section{Gut Microflora}

An higher coliforms bacteria colony count $(P<0.05)$ on gizzard was observed in groups $0.5 \mathrm{LB}$ and $1.5 \mathrm{LB}$ than in control (Table 4). In overall, more cecal enterococcus bacteria colonies $(P<0.05)$ were found when LB additive was supplemented in drinking water.

\section{Hematological, Lymphoid Organs and Humoral Immunity} Traits

Total plasma protein and albumin concentration were higher in group $0.5 \mathrm{LB}$ (3.45 and $1.47 \mathrm{~g} / \mathrm{d} l$, respectively) than control $(2.92$ and $1.20 \mathrm{~g} / \mathrm{d} l$, respectively; $P<0.05)$. A relative thymus weight improvement $(P<0.05)$ was observed in group 2.0LB when compared with control group (Table 5). However, the relative Bursa of Fabricius weight was lower $(P<0.05)$ in group 1.0LB than control group. The antibody titers against Avian Influenza disease and Infectious Bronchitis disease virus were not affected $(P>0.05)$ by LB supplementation in drinking water.

\section{Gut Gross Morphometry and Carcass Traits}

Left cecum diameter was lower in groups $0.5 \mathrm{LB}(0.27$ $\mathrm{mm})$ and 1.0LB $(0.26 \mathrm{~mm})$ than in control group $(0.35 \mathrm{~mm} ; P$ $<0.05)$. No other significant differences $(P>0.05)$ among groups on all viscera or carcass traits were observed.

\section{Discussion}

In present study, at the end of the production cycle daily feed, energy and proteins intakes, and live body weight or production indexes were similar between groups. These results are in agreement with similar total feed intake, final body weight observed by Marcinčáková et al. (2011a), in broilers fed with commercial diets and ground LB at $2 \%$ level. However, contrarily to our study, feed conversion ratio remained constant. Marcinčák et al. (2011b) also ob- 
Table 5. Lymphoid organs weight (g) and relative weight (\%)in broilers at 42nd day of age fed different levels of lemon balm extract into drinking water

\begin{tabular}{ccccccc}
\hline \hline Item & $\begin{array}{c}\text { Thymus } \\
(\mathrm{g})\end{array}$ & $\begin{array}{c}\text { Thymus } \\
(\%)\end{array}$ & $\begin{array}{c}\text { Spleen } \\
(\mathrm{g})\end{array}$ & $\begin{array}{c}\text { Spleen } \\
(\%)\end{array}$ & $\begin{array}{c}\text { Bursa } \\
\text { Fabricius } \\
(\mathrm{g})\end{array}$ & $\begin{array}{c}\text { Bursa } \\
\text { Fabricius } \\
(\%)\end{array}$ \\
\hline Control & $3.7^{\mathrm{a}}$ & $0.20^{\mathrm{a}}$ & 2.9 & $0.16^{\mathrm{ab}}$ & $4.9^{\mathrm{a}}$ & $0.26^{\mathrm{a}}$ \\
$0.5 \mathrm{LB}$ & $6.2^{\mathrm{ab}}$ & $0.31^{\mathrm{ab}}$ & 3.1 & $0.15^{\mathrm{ab}}$ & $4.2^{\mathrm{ab}}$ & $0.21^{\mathrm{ab}}$ \\
$1.0 \mathrm{LB}$ & $5.3^{\mathrm{ab}}$ & $0.28^{\mathrm{ab}}$ & 2.7 & $0.14^{\mathrm{ab}}$ & $2.8^{\mathrm{b}}$ & $0.15^{\mathrm{b}}$ \\
$1.5 \mathrm{LB}$ & $5.6^{\mathrm{ab}}$ & $0.30^{\mathrm{ab}}$ & 2.3 & $0.12^{\mathrm{a}}$ & $4.1^{\mathrm{ab}}$ & $0.22^{\mathrm{ab}}$ \\
$2.0 \mathrm{LB}$ & $7.1^{\mathrm{b}}$ & $0.41^{\mathrm{b}}$ & 3.2 & $0.18^{\mathrm{b}}$ & $3.7^{\mathrm{ab}}$ & $0.22^{\mathrm{ab}}$ \\
$( \pm$ SEM $)$ & $( \pm 0.5)$ & $( \pm 0.03)$ & $( \pm 0.2)$ & $( \pm 0.01)$ & $( \pm 0.3)$ & $( \pm 0.01)$ \\
\hline
\end{tabular}

LB - Lemon balm extract.

Different letters within the same column indicate significant differences among treatment groups $(P<$ $0.05)$.

served unchanged feed consumption, daily weight gain, feed conversion ratio and live body weight, at 42nd day, when $1 \%$ clove buds powder (Syzygium aromaticum L.) and 0.2\% LB were added to feed or water respectively. Similar broiler performance results were observed by Petroviv et al. (2011) using the same herbal combination. However, Kwiecieñ and Winiarska-Mieczan (2009) observed a higher live body weight at 42 nd day in broilers supplemented with dried $2 \%$ LB than in broilers fed with flavomycin (antimicrobial) and dried grass silage $(2 \%)$, suggesting a positive effect of the herbal on broiler performance.

Although in our study the feed, energy and protein efficiency at 42nd day, were lower in group 1.0LB than control group, greater LB level (2.0LB) seems to attenuate this effect. Additionally, in the first two weeks, during starter period, the daily weight gain was lower in groups $0.5 \mathrm{LB}, 1.0$ LB or $1.5 \mathrm{LB}$ than in control group. No other differences between groups were observed. The adverse or neutral effects of LB on performance during this early period can be related with the fact that the gut digestion development is only completed around the 3rd week of broiler life (Noy and Sklan, 1995).

Moreover, on the 3rd week the fed, energy and protein intake were improved in groups $0.5 \mathrm{LB}, 1.0 \mathrm{LB}$ and $2.0 \mathrm{LB}$ without efficiency degradation in the last two groups. These improvements, considering the control group as reference, persisted during the 5th and 6th weeks only for group 1.0LB. During 5th week, groups $0.5 \mathrm{LB}, 1.0 \mathrm{LB}$ and 2.0LB showed higher daily weigh gain than control group. In Petroviv et al. (2011) study, the live body weight remained similar between groups during each one of the six weeks. More studies are necessary in order to evaluate if the LB water or feed supplementation only in finisher period, or even in onset of the grower period, is advantageous for broilers performance.

In overall, our study showed that colony counts of total coliform, but not E. coli (some strains are pathogenic), on gizzard (groups $0.5 \mathrm{LB}$ and $1.5 \mathrm{LB}$ ) and enterococcus bacteria on cecum, but not on total lactic acid producing bacteria (groups 1.0LB, 1.5LB and 2.0LB) were improved by LB. Despite some pathogenic species such as Enterococcus faecalis and Enterococcus faecium (Tremblay et al., 2011), enterococcus are also lactic acid producing bacteria and can modulate the remaining microflora. In fact, the groups 0.5 LB and 1.0LB negatively affected the left cecum diameter, probably due to a villi underdevelopment, and both findings can be related. The gut microflora enhancement and low cecal development can justify, at least partially (Lu et al., 2003; Lan et al., 2005), the poorly indexes efficiency observed in the present study.

Other than total proteins and albumin plasma levels improvement by group $0.5 \mathrm{LB}$, no differences in hematological parameters were observed in the present study, and in agreement with Petroviv et al. (2011) results, suggesting that the LB don't affect apparent health status of broilers.

Based on our findings, the group 2.0LB improved the relative weight of thymus, responsible for $\mathrm{T}$ lymphocytes production (Le Douarin and Dieterlen-Lièvre, 2013). However, the group 1.0LB had an adverse effect on relative weight of Bursa Fabricius, a critical organ for the B lymphocytes development (Ratcliffe, 2006). Additionally, the tested antibody titers remained unchanged on all LB groups. These results indicate the difficulty to positively modulate cellular and humoral immunity only using LB additive.

The LB at different levels don't influenced the weight of broiler carcass parts. This results are in agreement with Marcinčák et al. (2011b) and by Eleroğlu et al. (2014) using Hubbard S757 and Hubbard Grey Barred JA slow-growing genotypes until 81 st day of organic broiler production and fed with 1\% LB in diet. Maślanko and Pisarski (2009) observed a similar (relative) weight of abdominal fat in chilled carcass between broilers fed with flavomycin as grown promotor and fed with $2 \%$ LB powder. However, a higher relative weight of leg and breast muscles were observed by Marcinčáková et al. (2011a) with $2 \%$ LB powder.

Finally, in order to emphasize the potential impact of LB use on broiler welfare production we also report that the survival rate of $100 \%$ during broiler grower period was observed by Skomorucha and Sosnówka-Czajka (2013) when $0.2 \% \mathrm{LB}$ alcoholic extract was added to drinking water from 21 st to 35 th day. These researchers also observed that 
the feed supplementation with a mixture of goat's rue (Galega officinalis L.), common nettle (Urtica dioica L.), LB and common sage (Salvia officinalis L.), in the last 7 days of production cycle, can alleviated the stress provoked by longer transport distances to slaughter up to $300 \mathrm{~km}$.

\section{Conclusions}

The present study suggested that the $\mathrm{LB}$ at $1.0 \mathrm{~mL} / \mathrm{L}$ level added to drinking water during whole production cycle had adverse effect on feed, energy and protein efficiency without weight gain advantages. However, in the finisher period, LB can improve feed, energy and protein intakes without affect health status or carcass gross morphometry. As consequence, dietary $1.0 \mathrm{~mL} / \mathrm{L} \mathrm{LB}$, only during finisher period can improve broiler performances. However, further researches are need in order to evaluate the potential positive effects of LB, with special emphasis for $0.1 \%$ and $0.2 \%$ levels, on birds performance, and also considering health status and welfare when supplemented only during the finisher period or even on grower period.

\section{Acknowledgments}

This manuscript is prepared from MSc. thesis of the first author. We are grateful to the Rasht Branch, Islamic Azad University, Rasht, Iran, and also University of Guilan, Rasht, Iran for supports.

\section{References}

Alzawqari MH, Al-Baddany AA, Al-Baadani HH, Alhidary IA, Khan RU, Aqil GM and Abdurab A. Effect of feeding dried sweet orange (Citrus sinensis) peel and lemon grass (Cymbopogon citratus) leaves on growth performance, carcass traits, serum metabolites and antioxidant status in broiler during the finisher phase. Environmental Science and Pollution Research 23: 17077-17082. 2016.

Bombik T, Bombik E, Frankowska A, Trawińska B and Saba L. Effect of herbal extracts on some haematological parameters of calves during rearing. Bulletin- Veterinary Institute in Pulawy, 56: 655-658. 2012.

Dhama K, Shyma K Latheef, Saminathan M, Abdul Samad H, Karthik K, Tiwari R, Khan RU, Alagawany M, Farag MR, Alam GM, Laudadio V and Tufarelli V. Multiple beneficial applications and modes of action of herbs in poultry health and production-A review. International Journal of Pharmacology, 11: 152-176. 2015.

Eleroğlu H, Yıldırım A, Işıklı ND, Şekeroğlu A and Duman M. Comparison of meat quality and fatty acid profile in slowgrowing chicken genotypes fed diets supplemented with Origanum vulgare or Melissa officinalis leaves under the organic system. Italian Journal of Animal Science, 12: e64. 2013.

Eleroğlu H, Yıldırım A, Şekeroğlu A and Duman M. Comparison of the growth performance and carcass characteristics of two slow-growing broiler genotypes fed diets supplemented with dry oregano (Origanum vulgare L.) or lemon balm (Melissa officinalis L.) leaves under the organic system. Kafkas Üniversitesi Veteriner Fakültesi Dergisi, 20: 49-58. 2014.

Kato-Noguchi H. Assessment of allelopathic potential of shoot powder of lemon balm. Scientia Horticulturae, 97: 419-423. 2003.
Khan RU, Naz S, Nikousefat Z, Tufarelli V, Javdani M, Qureshi MS and Laudadio V. Potential applications of ginger (Zingiber officinale) in poultry diets. World's Poultry Science Journal, 68: 245-252. 2012.

Kwiecieñ M and Winiarska-Mieczan A. Effect of addition of herbs on body weight and assessment of physical and chemical alterations in the tibia bones of broiler chickens. Journal of Elementology, 14: 705-715. 2009.

Lan Y, Verstegen MWA, Tamminga S and Williams BA. The role of the commensal gut microbial community in broiler chickens. World's Poultry Science Journal, 61: 95-104. 2005.

Le Douarin NM and Dieterlen-Lièvre F. How studies on the avian embryo have opened new avenues in the understanding of development: a view about the neural and hematopoietic systems. Development Growth \& Differentiation, 55: 1-14. 2013.

Lin J-T, Chen Y-C, Lee Y-C, Hou RC-W, Chen F-L and Yang D-J. Antioxidant, anti-proliferative and cyclooxygenase-2 inhibitory activities of ethanolic extracts from lemon balm (Melissa officinalis L.) leaves. LWT - Food Science and Technology, 49: 1-7. 2012

Lu J, Idris U, Harmon B, Hofacre C, Maurer JJ and Lee MD. Diversity and Succession of the Intestinal Bacterial Community of the Maturing Broiler Chicken. Applied Environmental Microbiology, 69: 6816-6824. 2003.

Marcinčák S, Mesarčová L, Popelka P, Čertík M, Šimková J, Marcinčáková D, Mala $\mathrm{P}$, Zachar $\mathrm{P}$ and Mártonová $\mathrm{M}$. The influence of dietary supplementation with Melissa officinalis and combination of Achillea millefolium and Crataegus oxyacantha on oxidative stability of stored poultry meat. Journal of Animal and Feed Science, 20: 236-245. 2011a.

Marcinčák S, Popelka P, Zdolec N, Mártonová M, Šimková J and Marcinčáková D. Effect of supplementation of phytogenic feed additives on performance parameters and meat quality of broiler chickens. Slovenian Veterinary Research, 48: 27-34. 2011b.

Marcinčáková D, Čertík M, Marcinčák S, Popelka P, Šimková J, Klempová T, Petrovič V, Tučková M and Bača M. Effect of dietary supplementation of Melissa officinalis and combination of Achillea millefolium and Crataegus oxyacantha on broiler growth performance, fatty acid composition and lipid oxidation of chicken meat. Italian Journal of Animal Science, 10: e43. 2011.

Maślanko W and Pisarski RK. The effect of herbs on the share of abdominal fat and its fatty acid profile in broiler chickens. Annales Universitatis Mariae Curie-Skłodowska, 27: 28-34. 2009.

Mimica-Dukic N, Bozin B, Sokovic M and Simin N. Antimicrobial and antioxidant activities of Melissa officinalis L. (Lamiaceae) essential oil. Journal of Agriculture and Food Chemistry, 52: 2485-2489. 2004.

Noy Y and Sklan D. Digestion and absorption in the young chick. Poultry Science, 74: 366-373. 1995.

Pereira RP, Boligon AA, Appel AS, Fachinetto R, Ceron CS, TanusSantos JE, Athayde ML and Rocha JBT. Chemical composition, antioxidant and anticholinesterase activity of Melissa officinalis. Industrial Crops and Products, 53: 34-45. 2014.

Petrovic V, Marcincak S, Popelka P, Simkova J, Martonova M, Buleca J, Marcincakova D, Tuckova M, Molnar L and Kovac G. The effect of supplementation of clove and agrimony or clove and lemon balm on growth performance, antioxidant status and selected indices of lipid profile of broiler chickens. 
Journal of Animal Physiology and Animal Nutrition, 96: 970977. 2012.

Ratcliffe MJ. Antibodies, immunoglobulin genes and the bursa of Fabricius in chicken B cell development. Developmental and Comparative Immunology, 30: 101-118. 2006.

Skomorucha I and Sosnówka-Czajka E. Effect of water supplementation with herbal extracts on broiler chicken welfare. Annals of
Animal Science, 13: 849-857. 2013.

Tremblay CL, Letellier A, Quessy S, Boulianne M, Daignault D and Archambault M. Multiple-antibiotic resistance of Enterococcus faecalis and Enterococcus faecium from cecal contents in broiler chicken and turkey flocks slaughtered in Canada and plasmid colocalization of tetO and ermB genes. Journal of Food Protection, 74: 1639-1648. 2011. 